\title{
An unresectable retroperitoneal malignant fibrous histiocytoma: A case report
}

\author{
PO-JEN HSIAO ${ }^{1}$, GUANG-HENG CHEN ${ }^{1}$, YI-HUEI CHANG ${ }^{1}$, CHAO-HSIANG CHANG $^{1}$, \\ $\mathrm{HAN} \mathrm{CHANG}^{2}$ and LI-YUAN BAI ${ }^{3,4}$ \\ Departments of ${ }^{1}$ Urology and ${ }^{2}$ Pathology, China Medical University Hospital; ${ }^{3}$ Division of Hematology and Oncology; \\ ${ }^{4}$ Department of Internal Medicine, China Medical University, Taichung 40447, Taiwan, R.O.C.
}

Received December 29, 2014; Accepted January 28, 2016

DOI: $10.3892 / 01.2016 .4283$

\begin{abstract}
Malignant fibrous histiocytoma (MFH) is most commonly observed in the extremities and the trunk but rarely in retroperitoneum. The present case report documents a 64-year-old man who was admitted with an abdominal palpable mass for 6 months. After a thorough investigation, a tumor of the retroperitoneum was identified adhered to adjacent organs and vessels. The patient experienced mild hydronephrosis and hydroureter as a result of the tumor compression. A number of previous surgeons considered the tumor unresectable and suggested palliative treatment. En bloc resection of the tumor was attempted but incomplete surgery was performed initially as the tumor was friable and prone to bleeding. Therefore, a biopsy of the tumor was performed and a double $\mathrm{J}$ ureteral stent was set for hydronephrosis. Histopathological examination confirmed the tumor was an $\mathrm{MFH}$. The patient received neo-adjuvant chemotherapy with 4 cycles of mesna, doxorubicin, ifosfamide, and dacarbazine (MAID). A computed tomography scan demonstrated that the tumor had reduced in size following chemotherapy. En bloc resection of the tumor was arranged again 6 months later. The tumor exhibited a complete response to neo-adjuvant chemotherapy after the formal pathological evaluation. The patient survives without tumor recurrence $>5$ years without recurrence.
\end{abstract}

\section{Introduction}

Malignant fibrous histiocytoma (MFH), which is also termed undifferentiated pleomorphic sarcoma or pleomorphic spindle cell sarcoma (PSCS), is a type of malignant sarcoma that occurs most frequently in patients aged between 50 and 70 years (1). MFH occurs most commonly in the extremities

Correspondence to: Dr Chao-Hsiang Chang, Department of Urology, China Medical University Hospital, 2 YuDe Road, Taichung 40447, Taiwan, R.O.C.

E-mail: urology8395@yahoo.com.tw

Key words: urologic neoplasms, retroperitoneal neoplasms, chemotherapy, Neo-adjuvant and the trunk, and it is extremely rare in the retroperitoneum $(1,2)$. The majority of retroperitoneal MFH cases are asymptomatic. Compression of nearby organs in the abdomen may elicit symptoms, including anorexia, abdominal discomfort, nausea and the sensation of an abdominal mass with abdominal girth enlargement (3). Chemotherapy is employed for advanced disease, however, large trials have not demonstrated a significant benefit (4,5). Recent insights into the neo-adjuvant chemotherapy of MFH provide exciting avenues for future research. A review of the clinical literature on this topic supports the management that was taken in the present case.

\section{Case report}

A 64-year-old male with an unremarkable medical history presented with a 6-month history of abdominal fullness at the China Medical University Hospital (Taichung, Taiwan) in April 2012. On abdominal physical examination, a painless mass was palpated in right abdomen. After thorough investigation, a tumor measuring $\sim 20 \times 12 \times 8 \mathrm{~cm}$ in size was identified in the retroperitoneum. The right internal and external iliac vessels were adhered to the tumor and medial deviation. Right mild hydronephrosis and hydroureter was observed in computed tomography (CT) scan due to external compression (Fig. 1).

The patient had previously sought medical opinion and palliative treatment was suggested by the majority of those consulted. Instead, the patient was admitted to our a tertiary referral hospital (China Medical University Hospital, Taichung, Taiwan) in April 2010. The patient underwent an exploratory laparotomy for removal of the tumor in May, 2012. The tumor was not completely removed due to a severe hemorrhage during the surgery when the tumor surface was approached. However, excisional biopsy of the tumor was performed and a double J stent for the right hydronephrosis was inserted.

A diagnosis of MFH (grade 3/3 based on the Fédération Nationale des Centres de Lutte Contre le Cancer soft tissue tumor grading system) was confirmed by a pathologist. Immunohistochemical analysis was performed by incubation with the following primary antibodies: Monoclonal mouse anti-human cytokeratin (CK; low molecular weight; AE1; dilution, 1:800; catalog no., Z2269; Zeta Corp., Sierra Madre, CA, USA), 


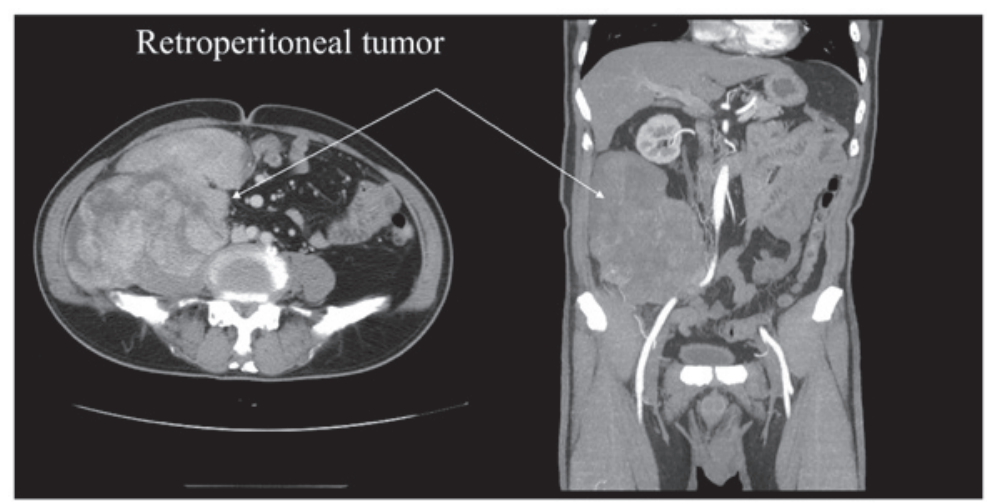

Figure 1. Abdominal computed tomography scan prior to mesna, tetrahydropyranyl adriamycin, ifosfamide and dacarbazine treatment. The tumor measured $\sim 20 \times 12 \times 8 \mathrm{~cm}$ and was situated in the retroperitoneum. The tumor caused right internal, external iliac arteries and right ureter medial displacement, mild hydronephrosis and hydroureter.

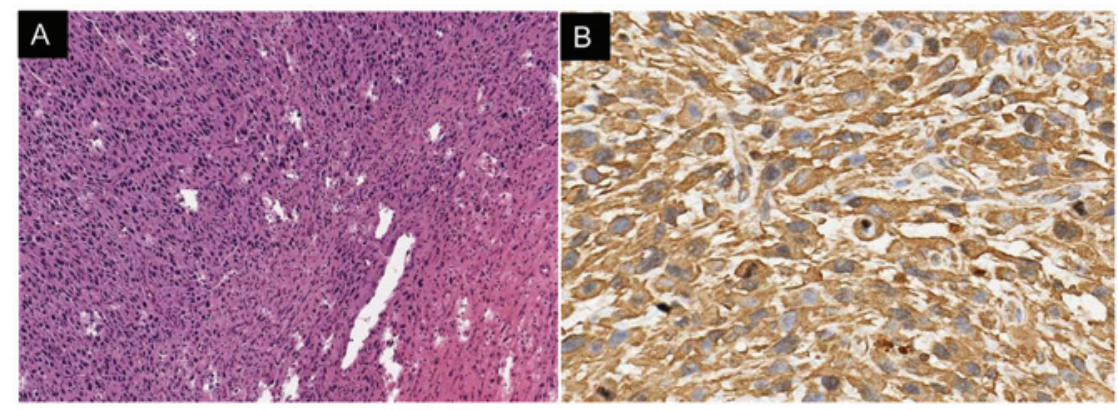

Figure 2. Microscopy and photomicrography of the tumor prior to chemotherapy. (A) Microscopic examination revealed the tumor was composed of a mixture of spindle cells in a storiform pattern and polygonal or rounded cells (hematoxylin and eosin staining; magnification, x100). (B) Immunohistochemical staining revealed the tumor cells were positive for vimentin (magnification, $\mathrm{x} 400$ ).

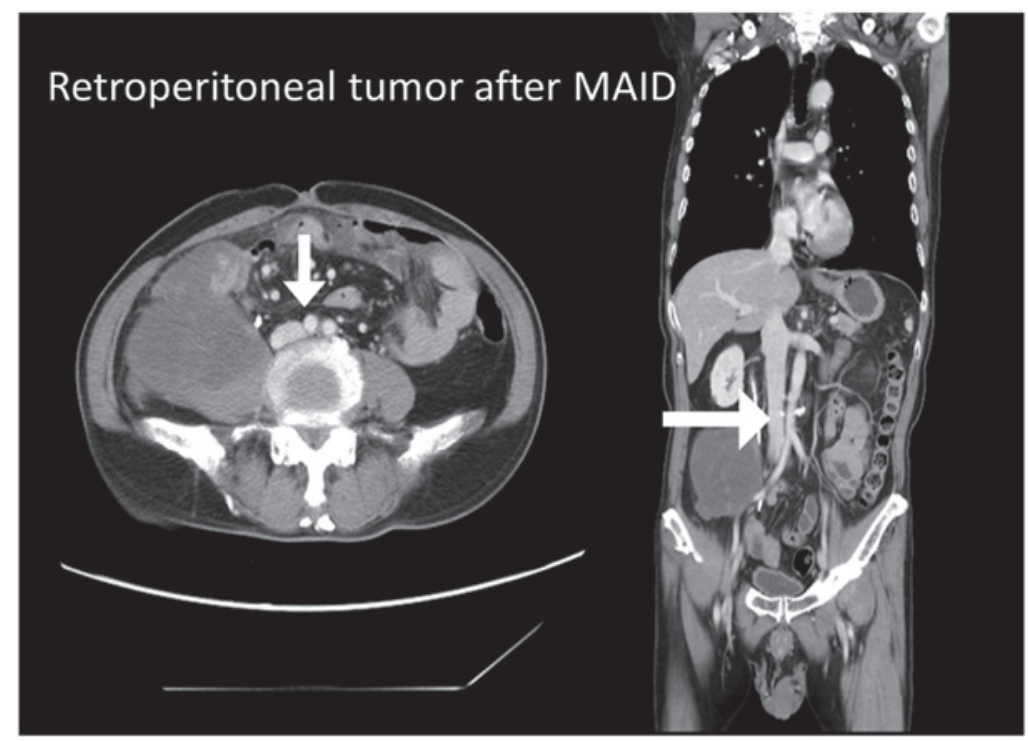

Figure 3. Abdominal scan following MAID treatment. The mass was decreased in size and had reduced levels of necrosis compared with the previous CT scan. White arrows indicate the inferior vena cava. CT, computed tomography; MAID, mesna, tetrahydropyranyl adriamycin, ifosfamide and dacarbazine.

monoclonal mouse anti-human CK (high molecular weight; AE3; dilution, 1:800; catalog no., Z2267; Zeta Corp.) monoclonal mouse anti-human smooth muscle actin (dilution, 1:100; catalog no., NCL-SMA; Leica Biosystems, Wetzlar, Germany), monoclonal mouse anti-human desmin (dilution, 1:200; catalog no., DES-DERII-CE; Leica Biosystems), monoclonal mouse anti-human vimentin (dilution, 1:400; catalog no., 61-0066; Genemed Biotechnologies, Inc., San Francisco, CA, USA), monoclonal mouse anti-human melan-A (dilution, 1:50; catalog no., NCL-MelanA; Leica Biosystems), monoclonal 


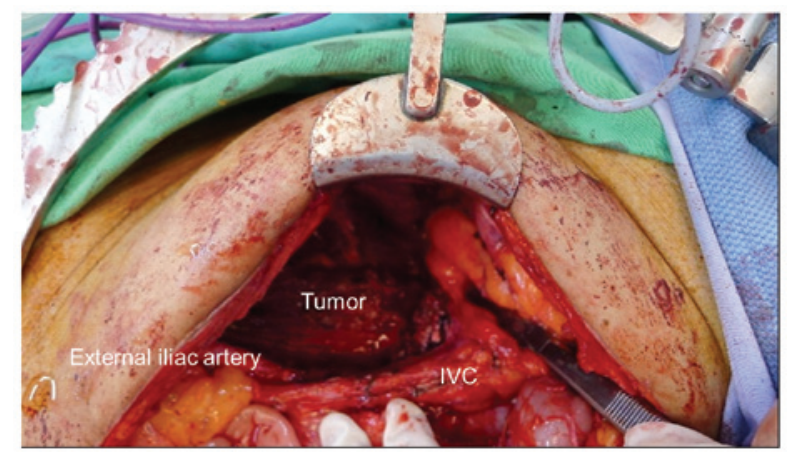

Figure 4. The tumor adhered to the right gonadal vein, adjacent to right common iliac, internal and external iliac arteries, anterior to the IVC and medial to the right ureter, below the right kidney and ascending colon.

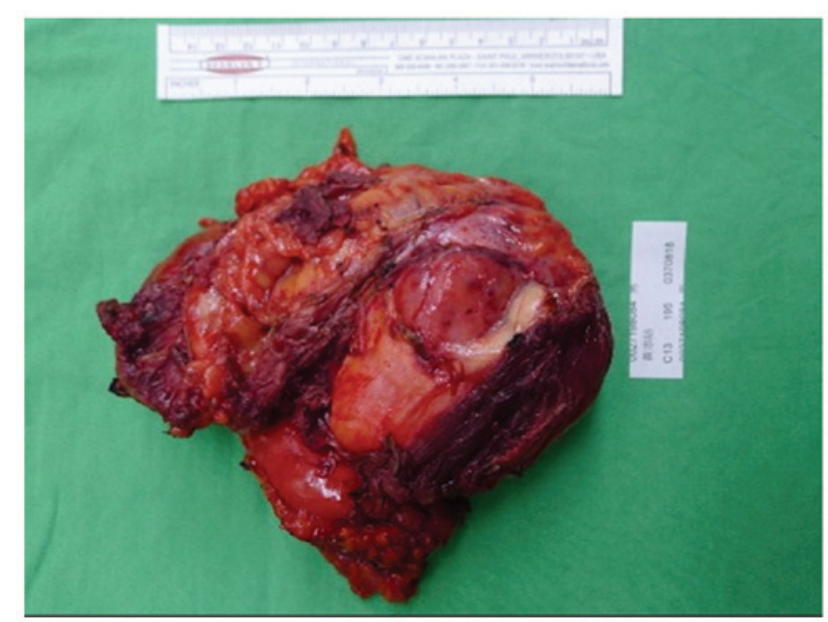

Figure 5. Complete resection of the tumor: The tumor size was $13.3 \times 8.8 \times 5.7 \mathrm{~cm}$.

rabbit anti-human S100 (1:400; catalog no, NCL-S100p; Leica Biosystems), monoclonal mouse anti-human cluster of differentiation (CD)68 (dilution, 1:800; catalog no., CD68-L-CE; Leica Biosystems) and monoclonal mouse anti-human HMB-45 (dilution, 1:100; catalog no., HMB45-L-CE; Leica Biosystems). Diaminobenzidine (Leica Biosystems) was used as a chromogen and Meyer's hematoxylin (Leica Biosystems) as a counterstain. An Olympus BX-50 microscope (Olympus Corp., Tokyo, Japan) with DP-20 digital camera (Olympus Corp.) was used to capture images at a magnification of x100 and magnification, x400 (vimentin immunostaining). Immunohistochemistry revealed positivity for vimentin; and negativity for CK, desmin, S-100 and CD117 (Fig. 2). Neo-adjuvant chemotherapy was suggested for palliative treatment. The patient received neo-adjuvant chemotherapy with 4 cycles of MAID [mesna 2,500 mg/m²/6 h (days 1-3), tetrahydropyranyl adriamycin $20 \mathrm{mg} / \mathrm{m}^{2} / 0.5 \mathrm{~h}$ (days 1-3), ifosfamide $2,500 \mathrm{mg} / \mathrm{m}^{2} / 6 \mathrm{~h}$ (days 1-3) and dacarbazine $300 \mathrm{mg} / \mathrm{m}^{2} / 1 \mathrm{~h}$ (days 1-3)] (6) from June to October 2012. CT scan was arranged after undergoing 2 cycles of chemotherapy. The mass was reduced in size (minor response) with central necrosis components in comparison to the last CT scan (Fig. 3). Six months later, the patient underwent whole tumor resection in November 2012. The tumor was carefully removed from the right gonadal vein, right common iliac, internal and external iliac arteries. (Fig. 4) The tumor had

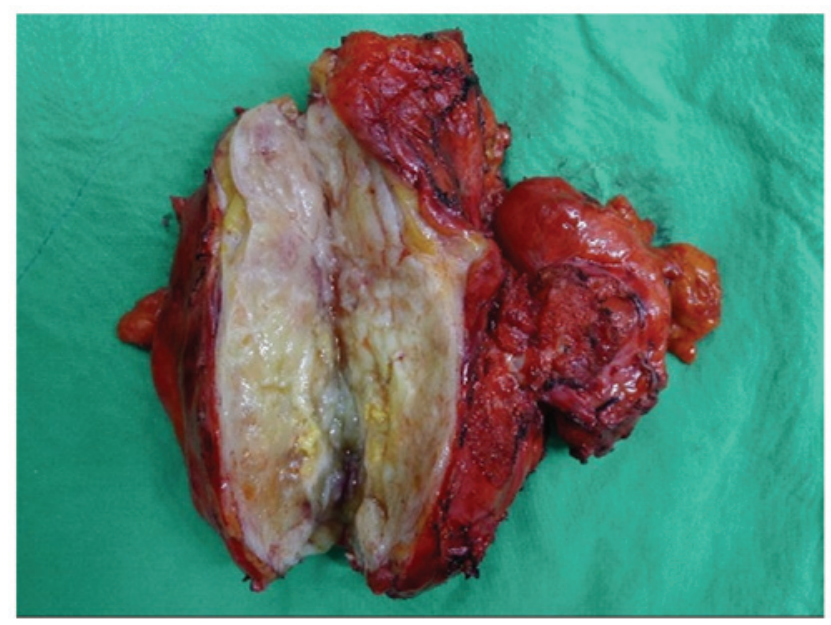

Figure 6. Dissection revealed that the interior of the tumor was a yellow-white color, with necrotic tissue.
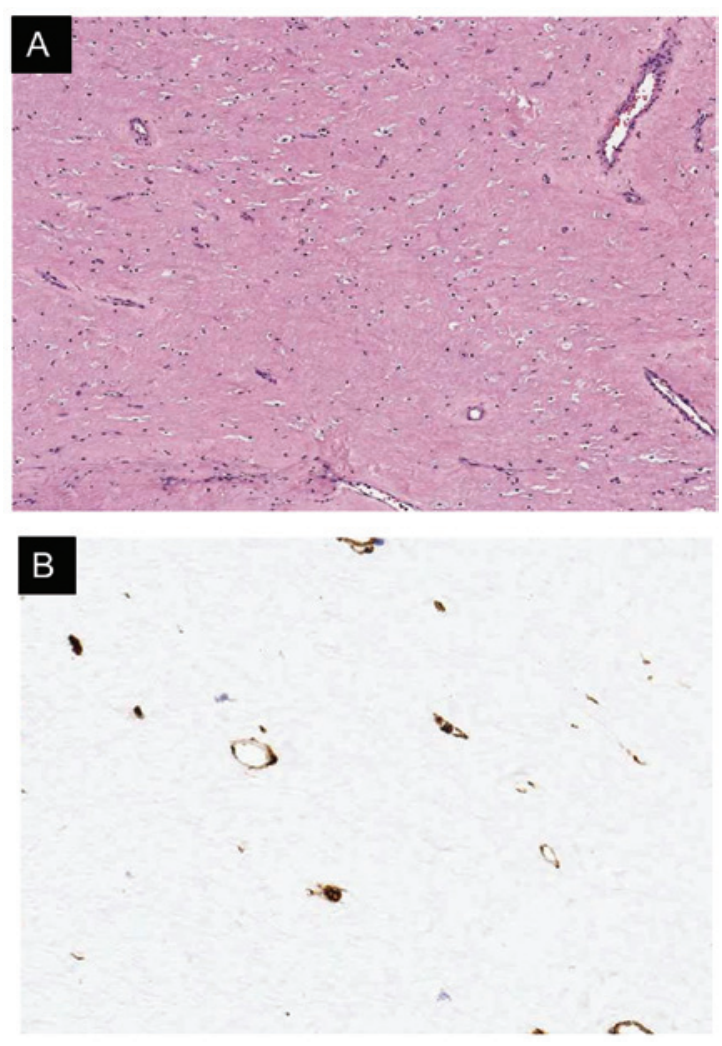

Figure 7. Microscopy and photomicrography of the tumor following chemotherapy. (A) Nuclear pleomorphism, tumor necrosis and degenerative foamy tumor cells with intracellular brown pigment deposition were present. Tumor cells responded well to chemotherapy with extensive tumor necrosis and fibrosis (hematoxylin and eosin staining; magnification, x100). (B) Immunohistochemical staining revealed that the vimentin positive tumor component was reduced following chemotherapy (magnification, $\mathrm{x} 400$ ).

also invaded the right psoas muscle and anterior longitudinal ligament of the lumbar spine. Part of the muscle and ligament were also therefore resected. En bloc resection was undergone successfully without any remaining tumor. The surgical specimen measured $13.3 \times 8.8 \times 5.7 \mathrm{~cm}$ in size and weighed $4.3 \mathrm{~kg}$. (Fig. 5) The specimen had heterogeneous yellowish content and necrotic components inside. Extensive tumor necrosis 
and fibrosis were noted microscopically (Fig. 6). Complete response of chemotherapy was observed following pathological review, where a pathological complete response was defined as $99-100 \%$ necrosis (7-9). Pathology composed of a mixture of spindle cells in a storiform pattern and polygonal or rounded cells. Nuclear pleomorphism and necrosis were present in the resected tumor. Degenerative foamy tumor cells with intracellular brown pigment deposition were observed inside the tumor. Tumor cells responded well to chemotherapy with extensive tumor necrosis and fibrosis. Immunohistochemistry demonstrated that the tumor cells were negative for cytokeratin, smooth muscle actin, desmin, S-100, and melan-A expression, but positive with diffuse cytoplasmic staining for vimentin, CD68, and HMB-45. The Fontana-Masson and iron stains were negative for intracellular pigments. The negative margin was also proven under microscopy (Fig. 7). The patient did not receive any further treatment and remained alive without tumor recurrence at the final follow-up appointment in February 2015.

\section{Discussion}

MFH was first described as soft-tissue sarcomas arising from fibroblasts and histiocytes in 1964 (5). MFH is generally divided into 5 histological types: Storiform-pleomorphic, myxoid, giant cell, angiomatoid and inflammatory subtypes (10). MFH presents with varied histology morphology, but the classic form is composed of spindle-shaped and round histiocytes arranged in storiform pattern as in the present case.

The primary treatment of retroperitoneal MFH is surgical resection (11). The main structures in the retroperitoneum are the aorta, vena cava, superior mesenteric vessels, celiac trunk, kidney, ureter and duodenum (12). Damage to these structures may cause severe post-operative complications such as hemorrhaging and fatalities (12). If the tumor is encased or adheres to these structures, the mortality and morbidity rate will be high during and following tumor excision surgery.

Although the 5-year survival rate of all MFH patients that receive surgical resection is $67.2 \%$ (13), the 5-year survival rate of patients with those unresectable MFH is $<10 \%$ (14). To the best of our knowledge, no studies regarding the management of a marginally resectable or unresectable retroperitoneal MFH have been published. Retroperitoneal MFH may be shrunk following an initial course of chemotherapy. Multimodal therapy for extremity sarcoma has provided an inference for the role of chemotherapy in retroperitoneal lesions (2). There are certain survival benefits if the tumor can be removed after chemotherapy (15). Overall, the arguments for neo-adjuvant therapy are reasonable.

A study reported $70 \%$ of 63 patients underwent complete surgery after neoadjuvant chemotherapy of ifosfamide, cisplatin, adriamycin and mitomycin. But $65 \%$ of patients had disease relapsed and $34 \%$ of patients died of metastasis within 30 months following up. Median survival of patients was 30 months and median relapse-free survival was 13 months (16).

A meta-analysis study reported a slight advantage of $4 \%$ overall survival for the use of adjuvant chemotherapy to decrease the risk of death and recurrence in patients with high-grade lesions $(17,18)$.
In the present study, a combination regimen of MAID was used for neoadjuvant chemotherapy. The regimen has been successful in neo-adjuvant programs for sarcomas of the extremities $(19,20)$ compared with historical controls, yet less data are available with regard to other soft tissue sites. Bui-Nguyen et al (21) designed a randomized control trial that patients who had sarcoma of extremities received 4 cycles of standard dose and high dose of MAID. Their 3-year overall survival rate was 49.4 and 32.7\%; the progression-free survival rate was 32.4 and $14.0 \%$, respectively. Other studies have also indicated the benefit of MAID regimen; pre-operative MAID is effective in advanced adult soft tissue sarcomas $(22,23)$. Elias et al $(24)$ demonstrated that MAID treatment is effective for retroperitoneal sarcoma: The overall response rate was $47 \%$ (10\% complete response CR). The majority of responses $(\sim 70 \%)$ were observed within 2 cycles, the median times to progression was 9 months; and the median survival was 16 months (24). Variations in chemosensitivity between different histological types have been observed (25). For example, response rates of $>50 \%$ have been reported for synovial sarcoma (26) Similarly, myxoid liposarcomas are considered to be significantly more responsive than the majority of soft tissue sarcoma, although the evidence remains controversial $(27,28)$ Limited data was reported for MFH type in retroperitoneal tumors. Therefore, further prospective randomized trials on special types of soft tissue sarcomas and on subtypes of MFH are needed in order to make a more conclusive evaluation.

In conclusion, palliative treatment is not the only option for a borderline resectable or unresectable MFH in retroperitoneum. The age and any comorbidity of the patient together with the histology of the tumor need to be taken into account. If the tumor is chemosensitive and adjacent to critical organs, chemotherapy may render the tumor suitable for radical surgery. However, patients with MFH exceeding $5 \mathrm{~cm}$ are at a significant risk of developing metastases. The present study provides evidence that neo-adjuvant chemotherapy for those high grading or unresectable retroperitoneal tumor may lead to the possibility to perform complete resection or an improvement in prognostic outcome.

\section{References}

1. Weiss SW and Goldblum JR (eds): Malignant fibrous histiocytoma (pleomorphic undifferentiated sarcoma). In: Enzinger and Weiss's Soft Tissue Tumors. 5th edition. Mosby, London, pp1161-1182, 2008.

2. Marchese R, Bufo P, Carrieri G and Bove G: Malignant fibrous histiocytoma of the kidney treated with nephrectomy and adjuvant radiotherapy: A case report. Case Rep Med 2010, 802026, pii 2010.

3. Bhavsar T, Saeed-Vafa D, Harbison S and Inniss S: Retroperitoneal cystic lymphangioma in an adult: A case report and review of the literature. World J Gastrointest Pathophysiol 1: 171-176 2010.

4. Tarján M, Cserni G and Szabó Z: Malignant fibrous histiocytoma of the kidney. Scand J Urol Nephrol 35: 518-520, 2001.

5. O'Brien JE and Stout AP: Malignant fibrous xanthomas Cancer 17: 1445-1455, 1964.

6. Anagnostopoulos G, Sakorafas GH, Grigoriadis K and Kostopoulos P: Malignant fibrous histiocytoma of the liver: A case report and review of the literature. Mt Sinai J Med 72: 50-52, 2005.

7. Eilber FC, Rosen G, Eckardt J, Forscher C, Nelson SD, Selch M, Dorey F and Eilber FR: Treatment-induced pathologic necrosis: A predictor of local recurrence and survival in patients receiving neoadjuvant therapy for high-grade extremity soft tissue sarcomas. J Clin Oncol 19: 3203-3209, 2001.

8. Canter RJ, Martinez SR, Tamurian RM, Wilton M, Li CS, Ryu J, Mak W, Monsky WL and Borys D: Radiographic and histologic response to neoadjuvant radiotherapy in patients with soft tissue sarcoma. Ann Surg Oncol 17: 2578-2584, 2010. 
9. Shah D, Borys D, Martinez SR, Li CS, Tamurian RM, Bold RJ, Monjazeb A and Canter R: Complete pathologic response to neoadjuvant radiotherapy is predictive of oncological outcome in patients with soft tissue sarcoma. Anticancer Res 32: 3911-3915, 2012.

10. Hassan I, Park SZ, Donohue JH, Nagorney DM, Kay PA, Nasciemento AG, Schleck CD and Ilstrup DM: Operative management of primary retroperitoneal sarcomas: A reappraisal of an institutional experience. Ann Surg 239: 244-250, 2004.

11. Tseng WW, Wang SC, Eichler CM, Warren RS and Nakakura EK Complete and safe resection of challenging retroperitoneal tumors: Anticipation of multi-organ and major vascular resection and use of adjunct procedures. World J Surg Oncol 9: 143, 2011.

12. Pezzi CM, Rawlings MS Jr, Esgro JJ, Pollock RE and Romsdahl MM: Prognostic factors in 277 patients with malignant fibrous histiocytoma. Cancer 69: 2098-2103, 1992.

13. Ogura K, Goto T, Imanishi J, Shinoda Y, Okuma T, Tsuda Y, Kobayashi H, Akiyama T, Hirata M, Yamamoto A and Kawano H: Neoadjuvant and adjuvant chemotherapy with modified mesna, adriamycin, ifosfamide, and dacarbazine (MAID) regimen for adult high-grade non-small round cell soft tissue sarcomas. Int J Clin Oncol 18: 170-176, 2013.

14. Sherman KL, Wayne JD, Chung J, Agulnik M, Attar S, Hayes JP, Laskin WB, Peabody TD, Bentrem DJ,Pollock RE and Bilimoria KY: Assessment of multimodality therapy use for extremity sarcoma in the United States. J Surg Oncol 109: 395-404, 2014.

15. Wendtner CM, Abdel-Rahman S, Krych M, Baumert J, Lindner LH, Baur A, Hiddeman W and Issels RD: Response to neoadjuvant chemotherapy combined with regional hyperthermia predicts long-term survival for adult patients with retroperitoneal and visceral high-risk soft tissue sarcomas. J Clinical Oncol 20: 3156-3164, 2002.

16. Mohagheghi MA, Sadighi S, Raafat J, Mosavi-Jarrahi AR, Seddigh Z and Ghaemi A: Neoadjuvant chemotherapy with ifosfamide, cisplatin, adriamycin and mitomycin (IMAP) for high risk adult soft tissue sarcomas. Acta Med Iran 47: 133-138, 2009

17. Gilbeau L, Kantor G, Stoeckle E, Lagarde P, Thomas L, Kind M, Richaud P, Coindre JM, Bonichon F and Bui BN: Surgical resection and radiotherapy for primary retroperitoneal soft tissue sarcoma. Radiother Oncol 65: 137-143, 2002.

18. Pervaiz N, Colterjohn N, Farrokhyar F, Tozer R, Figueredo A and Ghert M: A systematic meta-analysis of randomized controlled trials of adjuvant chemotherapy for localized resectable soft-tissue sarcoma. Cancer 113: 573-581, 2008.

19. Mullen JT, Kobayashi W, Wang JJ, Harmon DC, Choy E, Hornicek FJ, Rosenberg AE, Chen YL, Spiro IJ and DeLaney TF: Long-term follow-up of patients treated with neoadjuvant chemotherapy and radiotherapy for large, extremity soft tissue sarcomas. Cancer 118: 3758-3765, 2012.
20. Van Glabbeke M, van Oosterom AT, Oosterhuis JW, Mouridsen H, Crowther D, Somers R, Verweij J, Santoro A, Buesa J and Tursz T: Prognostic factors for the outcome of chemotherapy in advanced soft tissue sarcoma: An analysis of 2,185 patients treated with anthracycline-containing first-line regimens-a european organization for research and treatment of cancer soft tissue and bone sarcoma group study. J Clin Oncol 17: 150-157, 1999.

21. Bui-Nguyen B, Ray-Coquard I, Chevreau C, Penel N, Bay JO, Coindre JM, Cupissol D, Italiano A, Bonichon F, Lotz JP, et al: High-dose chemotherapy consolidation for chemosensitive advanced soft tissue sarcoma patients: An open-label, randomized controlled trial. Ann Oncol 23: 777-784, 2012.

22. Fayette J, Penel N, Chevreau C, Blay JY, Cupissol D, Thyss A, Guillemet C, Rios M, Rolland F, Fargeot P, et al: Phase III trial of standard versus dose-intensified doxorubicin, ifosfamide and dacarbazine (MAID) in the first-line treatment of metastatic and locally advanced soft tissue sarcoma. Invest New Drugs 27: 482-489, 2009.

23. Kasper B, Ouali M, van Glabbeke M, Blay JY, Bramwell VH, Woll PJ, Hohenberger P and Schöffski P: Prognostic factors in adolescents and young adults (AYA) with high risk soft tissue sarcoma (STS) treated by adjuvant chemotherapy: A study based on pooled European organisation for research and treatment of cancer (EORTC) clinical trials 62771 and 62931. Eur J Cancer 49: 449-456, 2013.

24. Elias A, Ryan L, Sulkes A, Collins J, Aisner J and Antman KH: Response to mesna, doxorubicin, ifosfamide, and dacarbazine in 108 patients with metastatic or unresectable sarcoma and no prior chemotherapy. J Clin Oncol 7: 1208-1216, 1989.

25. Salgado Rand vanMarck E: Soft tissue tumours: The surgical pathologist's perspective. In: Imaging of Soft Tissue Tumors. De Schepper AM, Vanhoemacker F, Gielen J and Parizel PM (eds). 3rd edition. Springer, Berlin, Germany, pp107-116, 2006.

26. Spurrell EL, Fisher C, Thomas JM and Judson IR: Prognostic factors in advanced synovial sarcoma: An analysis of 104 patients treated at the Royal Marsden Hospital. Ann Oncol 16: 437-444, 2005.

27. Karavasilis V, Seddon BM, Ashley S, Al-Muderis O, Fisher C and Judson I: Significant clinical benefit of first-line palliative chemotherapy in advanced soft-tissue sarcoma: Retrospective analysis and identification of prognostic factors in 488 patients. Cancer 112: 1585-1591, 2008.

28. Jones RL, Fisher C, Al-Muderis O and Judson IR: Differential sensitivity of liposarcoma subtypes to chemotherapy. Eur J Cancer 41: 2853-2860, 2005. 